\title{
L'IMMIGRATION SUBSAHARIENNE EN TUNISIE : DE LA RECONNAISSANCE D'UN FAIT SOCIAL À LA CRÉATION D'UN ENJEU GESTIONNAIRE
}

\author{
Camille Cassarini
}

Centre d'information et d'études sur les migrations internationales | « Migrations Société »

2020/1 N 179 | pages 43 à 57

ISSN 0995-7367

Article disponible en ligne à l'adresse :

https://www.cairn.info/revue-migrations-societe-2020-1-page-43.htm

Distribution électronique Cairn.info pour Centre d'information et d'études sur les migrations internationales.

(C) Centre d'information et d'études sur les migrations internationales. Tous droits réservés pour tous pays.

La reproduction ou représentation de cet article, notamment par photocopie, n'est autorisée que dans les limites des conditions générales d'utilisation du site ou, le cas échéant, des conditions générales de la licence souscrite par votre établissement. Toute autre reproduction ou représentation, en tout ou partie, sous quelque forme et de quelque manière que ce soit, est interdite sauf accord préalable et écrit de l'éditeur, en dehors des cas prévus par la législation en vigueur en France. Il est précisé que son stockage dans une base de données est également interdit. 


\title{
L'immigration subsaharienne en Tunisie : de la reconnaissance d'un fait social à la création d'un enjeu gestionnaire
}

\author{
Camille CASSARINI
}

\section{RÉSUMÉ}

L'immigration subsaharienne en Tunisie s'impose depuis 2011 comme nouveau fait social. Si les formes socio-spatiales de cette immigration sont multiples et concernent aussi bien des travailleurs que des étudiants, elle n'en reste pas moins cantonnée à une irrégularité juridique structurelle et à une mise sous silence de la part de l'État. Toutefois, ces populations font l'objet d'une politique de gestion sociale, construite et financée principalement par les organisations internationales et l'Union européenne et mise en ceuvre par un ensemble de partenaires humanitaires et associatifs actifs au niveau local. Cet article entend revenir sur les mécanismes ayant construit cette politique de gestion humanitaire et interroge les modalités de sa mise en ouvre. Il contribue ainsi à remettre en débat la "frontière » entretenue entre humanitaire et sécuritaire et propose, à partir du cas tunisien, une réflexion sur l'implication des acteurs humanitaires dans le processus d'externalisation des frontières en Afrique méditerranéenne.

MoTS-CLÉS : Africains noirs, aide humanitaire, OIM, politique migratoire, Tunisie, Union européenne

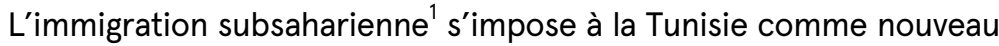
fait social. Qu'ils soient étudiants, travailleurs-migrants, aventuriers, réfugiés, demandeurs d'asile, tous sont aujourd'hui installés en Tunisie, pour des raisons et avec des projets différents. Ces individus partagent cependant la réalité indéniable et commune d'un enracinement de plus en plus profond et durable, même si parfois contraint, dans le territoire tunisien. Depuis 2011, ces immigrés deviennent de plus en plus visibles sous des formes de plus en plus structurées : dans le tissu associatif local, dans les associations communautaires ${ }^{2}$ et dans les églises et lieux de culte où ils participent d'une véritable revitalisation du fait religieux chrétien ${ }^{3}$. On les trouve aussi dans de très nombreux secteurs écono-

* Doctorant en géographie au sein du Laboratoire population environnement développement (LPED), Aix-Marseille Université.

1. Si la Tunisie est bien sûr un pays africain, le terme « immigration africaine » est ici employé d'un point de vue émique.

2. Par exemple: I'Association des Ivoiriens actifs de Sfax (AIVAS) ou encore I'Association des Ivoiriens actifs de Tunisie (ASSIVAT).

3. BOISSEVAIN, Katia, "Migrer et réveiller les Églises : diversification des cultes chrétiens en Tunisie", L'Année du Maghreb, n 11, 2014, pp. 105-121. 
miques où ils constituent une main-d'œuvre particulièrement recherchée. Complexe dans ses temporalités et multiforme, cette immigration se trouve porteuse et victime de stigmates à la fois pour ce qu'elle signifie pour la Tunisie (une nouvelle altérité ethnique, religieuse et culturelle), mais aussi pour ce qu'elle représente pour les pays européens, à travers le regard qu'ils portent sur la Tunisie (une «pression migratoire » qu'il faudrait à tout prix contenir dans un pays tiers). Ainsi, à rebours de deux idées dominantes, la première selon laquelle la situation de l'immigration subsaharienne ne serait liée qu'à la situation politique interne de la Tunisie, la seconde qui voudrait que tout phénomène migratoire dans les pays de la rive sud de la Méditerranée soit le fait et la conséquence des actions de l'Union européenne (UE), il est ici postulé qu'au contraire, la politique migratoire menée par la Tunisie à l'égard de ses immigrés est plutôt le produit de concurrences, de pressions exercées réciproquement et que le résultat concret de ces luttes aboutit à une forme négociée de politique de gestion migratoire convenant aux deux parties. Le second postulat qui est fait est que pour saisir au mieux les mécanismes producteurs de cette politique gestionnaire, il convient d'en appréhender les volets sécuritaire et humanitaire, non pas comme deux logiques antagonistes, mais bien comme deux logiques complémentaires qui, articulées l'une à l'autre, constituent la matrice dans laquelle se formule les actions mises en œuvre dans le domaine de la gestion de l'immigration. Cette matrice se caractérise donc par un assemblage de deux types de dispositifs. D'une part, des dispositifs sécuritaires et juridiques criminalisant l'immigration dans sa forme irrégulière (coopération des gardes-côtes tunisiens avec Frontex, loi du 3 février $2004^{4}$, pratiques policières coercitives, enfermement) et hérités du régime pré-2011. D'autre part, des dispositifs humanitaires de gestion des populations directement structurés par le migration management ou « approche globale des migrations $\gg^{5}$ promu et mis en œuvre en Tunisie par l'Organisation internationale pour les migrations (OIM) et ses partenaires ${ }^{6}$. Ces deux visions, I'une centrée sur l'idée que l'immigration représente un risque sécuritaire, l'autre s'appuyant sur une vision victimaire et misérabiliste des migrants, constituent les piliers sur lesquels repose la politique migratoire européo-tunisienne.

4. Voir https://www.jurisitetunisie.com/tunisie/codes/passeport/passeport2000.htm.

5. KABBANJI, Lama, "Migration et développement: quelles politiques menées en Afrique subsaharienne?", in : BEAUCHEMIN, Cris ; KABBANJI, Lama ; SAKHO, Papa ; SCHOUMAKER, Bruno (sous la direction de), Migrations africaines : le codéveloppement en questions, $\mathrm{Pa}-$ ris : Éd. Armand Colin, 2013, pp. 41-90.

6. En Tunisie, I'olm est partenaire de 28 institutions publiques, 46 organisations de la société civile, et cinq organisations internationales. Source : http://tunisia.iom.int/partenariatsoim-tunisie. 
II apparaît également difficile de traiter la question de l'immigration subsaharienne en Tunisie sans prendre en compte un facteur important de son occultation : la prépondérance dans l'espace politique et médiatique de la question des harragas ${ }^{7}$ tunisiens, qui concentre sur elle l'ensemble des interrogations et des débats produits autour de la question migratoire, en tout cas jusqu'à récemment. Ce premier facteur de compréhension est important, car il permet déjà d'esquisser un des principaux paradoxes de la question migratoire en Tunisie: si la société tunisienne semble se penser depuis les années 1970 comme terre d'émigration (1 223213 émigrés en $\left.2012^{8}\right)$, réclamant par là une série de droits en matière de liberté de circulation entre la Tunisie et I'Union européenne, elle a toujours beaucoup plus de difficultés à se penser comme terre d'immigration, et à assurer du même coup une série de droits à ses populations immigrées. Précisons-le d'emblée, cet article ne traitera pas de cet aspect si vaste. II convient toutefois de garder à l'esprit que l'émigration et l'immigration, particulièrement dans le contexte tunisien, doivent être considérées comme les deux faces de la gestion des migrations.

Cet article entend traiter de l'émergence de la question de l'immigration africaine en Tunisie comme objet scientifique et comme enjeu d'une politique de gestion s'étant construite par à-coups. Dans un premier temps, il conviendra de restituer les temporalités sociospatiales dans lesquelles cette immigration s'est construite comme objet scientifique, mais aussi sociétal. Dans un second temps, il sera abordé sa constitution, à partir de 2011, en tant qu'enjeu gestionnaire, d'une part pour l'État tunisien mais aussi et surtout, pour les organisations intergouvernementales présentes en Tunisie. Enfin, dans un troisième temps, il sera proposé quelques éléments définitoires de la politique et des mécanismes de gestion de l'immigration en Tunisie, à travers l'analyse de la construction d'une configuration humanitaire structurée autour de dispositifs d'assistance et d'accompagnement.

Ce travail s'appuie sur une enquête de terrain menée de janvier 2016 à mai 2019 dans les villes de Sfax, lieu principal d'enquête, Médenine et Tunis. Cette enquête s'est composée de deux dispositifs ethnographiques regroupant 74 entretiens libres et semi-directifs, réalisés avec des individus subsahariens en migration, des travailleurs et responsables d'associations, d'organisations humanitaires non gouvernementales (ONG), intergouvernementales, des hommes et des femmes

7. Ce terme, en dialecte maghrébin, désigne les candidats à une émigration clandestine vers I'Europe.

8. Source: Office des Tunisiens de l'étranger, http://ote.nat.tn/wpcontent/uploads/20 18/05/Repartition_de_la_communaute_tunisienne_a_I_etranger_-2012.pdf. 
d'Église ainsi que des fonctionnaires tunisiens en charge des questions migratoires. Le principal matériau mobilisé pour cet article provient de notes de terrains, d'entretiens et d'observations. Peu d'entretiens sont cités et mentionnés afin de garantir l'anonymat des personnes qui ont accepté de témoigner.

\section{L'émergence d'une réalité africaine en Tunisie}

Pour beaucoup, l'émergence de la Tunisie comme pays d'immigration se situe en 2011 avec la forte médiatisation de la situation dans le camp de réfugiés de Choucha. Si la gestion dans l'urgence de cet exil forcé pouvait justifier à certains égards le traitement exceptionnel qui lui a été réservé, rappelons tout de même que ce camp n'était pas composé uniquement de ressortissants africains ${ }^{9}$. Ses occupants étaient aussi bien originaires de pays d'Afrique de l'Ouest et subsaharienne que de la péninsule indienne. L'intervention des organisations internationales $a$, dans les faits, permis à une très large majorité de ses occupants de rejoindre leur pays d'origine via l'importante mise en œuvre du dispositif «d'aide au retour volontaire $\gg^{10}$. Par ailleurs, ce camp, très isolé du point de vue spatial, se situait sur une zone militaire, n'était pas «ouvert » à tous et faisait l'objet d'une importante surveillance et d'un contrôle important. II ne s'y est pas développé une économie informelle directement intégrée à la société locale. Le camp de Choucha n'a que très tardivement fermé et entre 50 et 80 ressortissants africains en situation irrégulière y sont restés jusqu'en 2016, littéralement abandonnés par les autorités ${ }^{11}$ et ne subsistant qu'avec des dons distribués de manière très aléatoire par l'Organisation internationale pour les migrations ${ }^{12}$. Ainsi, le camp de Choucha n'a pas été le «déclencheur » d'une immigration massive de ressortissants africains. Aucune preuve n'existe quant à l'installation durable de ses

9. DOURGNON, Paul : KASSAR, Hassène, "Refugees in and out North Africa: A Study of the Choucha Refugee Camp in Tunisia", The European Journal of Public Health, Vol. 24, No. 1, 2014, pp. 6-10.

10. HAON, Nicanor, "Tunisie : pas de printemps pour les migrants", Plein droit, $n^{\circ} 94$, octobre 2012, pp. 31-35; BOUBAKRI, Hassan, "Migration et asile en Tunisie depuis 2011 : vers de nouvelles figures migratoires?", Revue européenne des migrations internationales, vol. 31, $n^{\circ} 3-4,2015$, pp. 17-39; MOTTET, Aurore, "Répartition et circulation : les enjeux de la catégorisation dans le camp de Choucha (Tunisie)", Critique internationale, vol. 72, n 3, 2016, pp. $21-34$; GARELLI, Glenda ; TAZZIOLI, Martina, Tunisia as a Revolutionized Space of Migration, New York: Palgrave Macmillan, 2017, 105 p.

11. TAZZIOLI, Martina, "'People not of our Concern': Rejected Refugees in Tunisia", Radical Philosophy, No. 184, 2014, pp. 2-6.

12. Pour plus de précisions, lire le communiqué du Forum tunisien pour les droits économiques et sociaux (FTDES) : https://www.fidh.org/fr/themes/droits-des-migrants/tunisie-eva cuation-du-camp-de-choucha-par-la-force. 
occupants dans les villes tunisiennes, et les chiffres officiels font état de très importants retours dans les pays d'origine.

Ce camp n'a été que le révélateur, du fait de son importante médiatisation, d'une réalité migratoire qui avait commencé à se structurer sur le territoire tunisien une dizaine d'années auparavant. En effet, sans remonter jusqu'aux années 1970, où la Tunisie accueillait certains hauts cadres des administrations de pays africains venus se former ${ }^{13}, c^{\prime}$ est véritablement l'installation du siège de la Banque africaine de développement à Tunis en septembre $2003^{14}$ qui a constitué le point de départ d'une immigration africaine, principalement ivoirienne, par le biais de réseaux qui se sont sédimentés à Tunis, puis dans les autres grandes agglomérations littorales, principalement à Sfax, dans la décennie qui a suivi ${ }^{15}$.

L'arrivée de ces cadres avec leur famille et personnel, la tenue contingente de la coupe d'Afrique des nations, ont dispersé sur le territoire de nombreux ressortissants africains, principalement ivoiriens, mais aussi camerounais et congolais, qui se sont par la suite constitués en réseaux migratoires très bien organisés et encore actifs aujourd'hui. Notons aussi que la période des années 2000 est un moment où la privatisation de l'enseignement supérieur tunisien est engagée dans une stratégie de développement consistant en une internationalisation axée en direction de l'Afrique de l'Ouest et subsaharienne ${ }^{16}$. C'est ce qui explique l'émergence d'une réalité étudiante assez rapidement institutionnalisée et légitimée par les autorités tunisiennes, notamment par la création et la reconnaissance de I'Association des étudiants et stagiaires africains en Tunisie (AESAT), interlocuteur historique de l'État dans le domaine migratoire.

Dès 2005, Hassan Boubakri et Sylvie Mazzella soulignaient la présence de plus en plus importante de migrants subsahariens dans la capitale tunisienne, partagée entre différents mondes, «étudiant», «travailleur» et «aventurier», cohabitant et fonctionnant sur des principes d'entraide toutefois relatifs. S'il existait bien une «migration

13. PELLICANI, Michela C. ; PALMISANO, Leonardo, "Le cas des migrations élitaires en Tunisie dans le cadre des migrations sud-sud", Statistica, vol. LXII, n³, 2002, pp. 553-569.

14. MAZZELLA, Sylvie, "Une minorité visible : les cadres subsahariens de la BAD en Tunisie", in : POUESSEL, Stéphanie (sous la direction de), Noirs au Maghreb : enjeux identitaires, Paris: Éd. Khartala, 2012, $167 \mathrm{p}$.

15. BOUBAKRI, Hassen; MAZZELLA, Sylvie, "La Tunisie entre transit et immigration : politiques migratoires et conditions d'accueil des migrants africains à Tunis", Autrepart, vol. $36, \mathrm{n}^{\circ} 4$, 2005, pp. 149-165.

16. MAZZELLA, Sylvie, "Étudiants africains dans les universités privées tunisiennes : nouvelle figure de l'étudiant 'international'", in : MAZZELLA, Sylvie (sous la direction de), La mondialisation étudiante. Le Maghreb entre Nord et Sud, Paris : Éd Khartala, 2009, pp. 327-347. 
d'aventure », celle-ci était toutefois cantonnée à un statut transitoire dans lequel la Tunisie ne tenait finalement qu'une place résiduelle et directement liée à sa proximité avec les côtes italiennes. Par ailleurs, si ce premier travail de recherche permet déjà de comprendre que l'immigration africaine en Tunisie était bien une réalité sociale, des interrogations subsistaient quant à la nature de son ancrage socioterritorial et au statut de la Tunisie, entre «pays d'immigration et pays de transit $\gg$. Cette tension statutaire sera profondément structurante pour l'ensemble des travaux qui seront produits sur cette question par la suite. En effet, différencier une «migration de travail » d'une «migration d'aventure » ou « de transit » relève d'une certaine subjectivité, et sert, par rebond, des intérêts tout à fait distincts. Les travaux produits sur la notion de «transit $\gg^{17}$ montrent aujourd'hui que son utilisation a pu conforter, voire servir d'élément justificatif, d'une part, à la mise en place des dispositifs d'externalisation des frontières par I'Union européenne dans les pays tiers, et d'autre part, à la perpétuation de politiques de gestion répressives du fait migratoire dans ces pays, qui ont, par ailleurs, d'importants passifs autoritaires ${ }^{18}$. Cette notion a plus globalement alimenté le «mythe de l'invasion $\gg^{19}$ selon lequel l'immigration subsaharienne au Maghreb n'était finalement que contrainte et uniquement dirigée vers l'Union européenne, alors même que seuls $10 \%$ de ces migrants tentaient une traversée ${ }^{20}$. C'est donc pour toutes ces raisons que la Tunisie s'est longtemps complue dans son statut de pays de «transit» alors même que les temporalités migratoires s'étiraient déjà sur plusieurs années pour de nombreux ressortissants subsahariens. II faudra attendre 2011 et la révolution tunisienne pour qu'un nouveau discours émerge sur la question de l'immigration africaine. Sans abandonner l'idée que la Tunisie est un pays de transit, de plus en plus de voix font émerger une autre réalité, basée cette fois sur le constat que cette immigration est maintenant ancrée et qu'elle nécessite la mise en place de dispositifs particuliers.

17. BREDELOUP, Sylvie, "Les temps du transit dans la migration africaine", Journal des africanistes, vol. 83, $\mathrm{n}^{\circ} 2,2013$, pp. 58-90.

18. BENSAAD, Ali, "Le déplacement des frontières vers le Sud", Projet, $n^{\circ} 302$, janvier 2008 , pp. 50-55.

19. DE HAAS, Hein, Le mythe de l'invasion: migration irrégulière d'Afrique de l'Ouest au Maghreb et en Union européenne [En ligne], Travail de recherche de l'IMI, octobre 2007, https://www.imi-n.org/publications/le-mythe-de-linvasion.

20. LESSAULT, David; BEAUCHEMIN, Cris, "Ni invasion, ni exode. Regards statistiques sur les migrations d'Afrique subsaharienne", Revue européenne des migrations internationales, vol. $25, n^{\circ} 1,2009$, pp. 163-194. 


\section{La construction de l'immigration comme enjeu gestionnaire}

La révolution tunisienne représente un bouleversement considérable du point de vue sociétal. L'irruption de nouvelles libertés publiques, la libération de la parole, le regard jeté sur des années de fonctionnement autoritaire font émerger de manière cathartique de nouveaux enjeux. II serait toutefois exagéré de dire que la reconnaissance de l'immigration a connu le même engouement. Toutefois, comme une certaine forme de rupture avec la gestion autoritaire du fait migratoire, les évènements de Choucha font émerger une face jusqu'alors inconnue de celle-ci, celle d'une gestion humanitaire. Dans les faits, l'arrivée massive de réfugiés dans la région frontalière avec la Libye laisse l'État tunisien complètement démuni. II n'existait aucune tradition d'accueil d'urgence à cette époque, hormis celle du Croissant-Rouge tunisien (CRT), et dans une moindre mesure, celle des missionnaires présents en Afrique et des organisations catholiques. Les institutions internationales « expertes » comme le Haut Commissariat des Nations unies pour les réfugiés (HCR) et I'OIM, même si elles étaient présentes en Tunisie depuis un certain temps, ne disposaient de bureaux que dans la capitale et leurs déplacements étaient soumis à de nombreuses contraintes $^{21}$. De ce fait, leurs actions dans le domaine migratoire avaient toujours été tout à fait marginales et étroitement contrôlées par le régime. C'est dans ces conditions que l'État a dû faire appel à ces deux organisations pour venir en aide aux réfugiés de Choucha. II est difficile de savoir dans quelle mesure cet évènement a pu jouer dans les relations qu'entretenaient l'État tunisien et I'Union européenne dans le domaine migratoire. De toute évidence, si l'ouverture démocratique et politique expérimentée par la Tunisie post-révolutionnaire fut fortement saluée et encouragée par I'UE, la question de l'immigration a connu elle, une profonde inertie, tant au niveau des pratiques que de sa conception par l'État tunisien et I'Union européenne ${ }^{22}$. Ne rompant que partiellement avec les méthodes coercitives et répressives qui étaient au fondement de la gestion migratoire de la Tunisie, la période post-révolutionnaire ouvre cependant la voie à un nouvel acteur gestionnaire : I'Organisation internationale pour les migrations.

Les raisons de l'augmentation des moyens et des actions de l'olm en Tunisie sont multiples. Les premières sont à chercher dans un contexte purement national. Nous pouvons tout d'abord avancer le fait que l'État

21. BADALIČ, Vasja, "Tunisia's Role in the EU External Migration Policy: Crimmigration Law, Illegal Practices, and Their Impact on Human Rights", Journal of International Migration and Integration, Vol. 20, $\mathrm{n}^{\circ} 1,2019$, pp. 85-100.

22. GEISSER, Vincent, "Tunisie, des migrants subsahariens toujours exclus du rêve démocratique", Migrations société, vol. 31, n 177, juillet-septembre 2019, pp. 3-18. 
tunisien ne s'est jamais véritablement intéressé à cette question et ne disposait donc d'aucune expertise dans le domaine du «migration management $\gg^{23}$. Surtout, dans une situation économique et sociale particulièrement instable, il est certain que l'État post-révolutionnaire n'avait ni les moyens ni l'envie de prendre à bras-le-corps la question de l'immigration et de se lancer dans une politique de régularisation des migrants subsahariens en situation irrégulière, à l'image de celle du Maroc. Certains fonctionnaires nous ont par ailleurs confié avoir peur qu'une telle politique produise un « appel d'air » et que la Tunisie ne se retrouve «assaillie ${ }^{24}$ de ressortissants subsahariens : «Jusqu'à maintenant, les autorités n'ont pas pris de mesures pour ça. I/s sont en situation irrégulière, ils doivent quitter le territoire. Point. Nous n'avons même pas à réfléchir à ça, puisque ceux qui sont ici veulent rejoindre les côtes européennes... [...] Regardez le Maroc, ils ont fait des régularisations et maintenant, ils en ont deux fois plus qu'avant $\gg^{25}$.

$D^{\prime}$ autres raisons à prendre en compte sont purement politiques. Face à un taux de chômage proche des $16 \%$ de la population active, une politique d'immigration relativement ouverte ne trouverait pas écho dans le champ politique tunisien. En définitive, aucun homme politique tunisien n'aurait intérêt à défendre une telle mesure et c'est ce qui explique par ailleurs l'absence de ce débat lors des différentes échéances électorales ${ }^{26}$.

En tout état de cause, il serait présomptueux de dire que l'État tunisien craint cette immigration et qu'il chercherait, à l'image de l'Algérie, à la réprimer en pratiquant massivement l'expulsion aux frontières. Aucune donnée ne permet d'affirmer que ces méthodes sont pratiquées de manière structurelle, malgré quelques cas particulièrement médiatisés ${ }^{27}$. Au contraire, les données récoltées auprès de migrants ivoiriens vivant en Tunisie depuis plusieurs années montrent que cette immigration s'est plutôt intégrée au tissu économique local, principa-

23. GEIGER, Martin ; PÉCOUD, Antoine (eds), The Politics of International Migration Management, London: Palgrave Macmillan, 2010, 305 p. ; BARTELS, Inken, "Practices and Power of Knowledge Dissemination", Movements, vol. 4, n¹, 2018, pp. 47-66.

24. Entretien réalisé avec un responsable du gouvernorat de Sfax en juin 2018.

25. Entretien réalisé avec un haut fonctionnaire en charge des questions migratoires au sein du ministère des Affaires sociales à Tunis en mai 2019.

26. Hormis la question de l'asile qui, conformément aux engagements internationaux de la Tunisie, a fait l'objet d'un projet de loi, toujours pas ratifié par le Parlement.

27. Lire à ce sujet AMRAOUI, Amal, "Réfugiés en Tunisie, des vies suspendues" [En ligne], Nawaat.org, 24 septembre 2015, https://nawaat.org/portail/2015/09/24/refugies-entunisie-des-vies-suspendues/; SBOUAI, Sana, "Des migrants expulsés à la frontière algérienne" [En ligne], Inkyfada.com, ${ }^{\text {er }}$ septembre 2015, https://inkyfada.com/fr/20 15/09/01/expulse-frontiere-migrant-algerie-ouardiya-tunisie/. 
lement dans les emplois les plus précaires et pénibles ${ }^{28}$ et fait l'objet d'une relative tolérance de la part des autorités. Ces migrants, s'ils sont discrets, n'en constituent pas moins une main-d'œuvre prête à occuper tous les types d'emplois, à des salaires inférieurs d'environ $30 \%$ à ceux des Tunisiens. Ainsi, cette immigration constitue un levier de compétitivité considérable pour l'économie tunisienne, tournée vers l'exportation. De ce fait, l'État tunisien n'aurait aucun intérêt à «reconnaître » le phénomène et à légiférer sur la question. C'est ce qui justifie le fait que l'État persévère à considérer l'immigration comme un phénomène résiduel qui, dans sa forme irrégulière, convient de facto à tous les acteurs concernés par sa présence: entrepreneurs, institutions publiques, etc.

D'autres raisons encore sont à chercher du côté des relations qu'entretiennent la Tunisie et l'Union européenne dans le domaine des migrations. La Tunisie n'est pas le point aveugle de la politique d'externalisation des frontières en Afrique méditerranéenne, bien au contraire $^{29}$. Elle est signataire de la quasi-totalité des traités et accords réalisés dans le domaine de la gestion des migrations ces vingt dernières années ${ }^{30}$. Elle est aussi un des principaux bénéficiaires des fonds débloqués depuis 2015 à l'issue du Sommet de la Valette. Ses obligations en font donc un des acteurs centraux de la lutte contre l'immigration irrégulière en Méditerranée. Plusieurs travaux ont spécifiquement abordé cette question, du point de vue purement juridique ${ }^{31}$, en prenant appui sur les transformations qu'a subi la législation tunisienne en matière de migration (loi de 2004). Dans le domaine du développement, la participation de la Tunisie à la lutte contre la migration irrégulière s'est véritablement imposée comme «conditionnalité à la coopération ». La plupart de ces accords et traités, s'ils restent

28. Lire à ce sujet l'étude : LABIDI, Lassaad; BENNOUR, Lotfi, L'emploi formel et informel des travailleurs immigrés en Tunisie : cartographie et profils socioéconomiques des travailleurs immigrés en Tunisie [En ligne], mai 2017, http://www.migration.nat.tn/images/pdf/etudesur-I-emploi-formel.pdf\#pdfjs.action=download.

29. CASSARINO, Jean-Pierre, "Channelled Policy Transfers: EU-Tunisia Interactions on Migration Matters", European Journal of Migration and Law, Vol. 16, n¹, 2014, pp. 97-123; CASSARINO, Jean-Pierre, "Réadmission des migrants : les faux-semblants des partenariats euro-africains", Politique étrangère, printemps 2016, pp. 25-33 ; PERRIN, Delphine, "Immigration et création juridique au Maghreb. La fragmentation des mondes et des droits", in : BENSAAD, Ali (sous la direction de), Le Maghreb à l'épreuve des migrations subsahariennes. Immigration sur émigration, Paris : Éd. Karthala, 2009, pp. 245-265.

30. Programme commun pour les migrations et la mobilité (РСMM), Processus de Khartoum, Processus de Rabat.

31. BEN ACHOUR, Souhayma; BEN JEMIA, Monia, Révolution tunisienne et migration clandestine vers l'Europe: réactions européennes et tunisiennes, Florence : Institut universitaire européen, 2011, 13 p. ; BEN JEMIA, Monia, "La Tunisie, cerbère des frontières européennes", Plein droit, $\mathrm{n}^{\circ} 73$, juillet 2007, pp. 35-38; BEN JEMIA, Monia, Le droit tunisien de I'immigration, Florence : Institut universitaire européen, 2011, 22 p. 
parfois flous sur leur mise en œuvre, concernent principalement les migrants tunisiens, qui représentent de loin leur «population cible» du fait de son importance dans les débarquements sur les côtes italiennes ${ }^{32}$, mais ils concernent aussi l'immigration ayant cours en Tunisie qui, si elle n'est jamais dénommée comme telle, concerne factuellement les populations africaines. Ainsi, si celles-ci ne sont pas l'objet central de ces accords, elles en constituent tout de même une dimension importante. À titre d'exemple, en juin 2018, sur les 180 migrants que comptait un bateau qui a fait naufrage au large de l'île de Kerkennah, $30 \%$ étaient des ressortissants de pays d'Afrique subsaharienne ${ }^{33}$, dont une majorité d'Ivoiriens. Les autorités locales, par exemple à Sfax, reconnaissent considérer la majorité des ressortissants subsahariens comme de potentiels candidats au départ.

\section{L'immigration en Tunisie comme « état d'exception 》}

Pour comprendre la montée en puissance de l'approche gestionnaire des migrations en Tunisie à l'égard de l'immigration africaine, il apparaît nécessaire de repréciser certains éléments de contexte ayant justement permis à cette approche de s'imposer comme «grille de lecture naturelle » et partagée par les acteurs légitimement reconnus dans le domaine. Hormis les pratiques répressives de l'État en la matière, la question migratoire $n^{\prime}$ avait jamais fait l'objet de pratiques humanitaires partagées par un «champ social » identifié comme tel, c'est-à-dire qu'aucune organisation, association ou institution n'avait véritablement «pensé » cette question et $n$ 'avait construit autour d'elle des réseaux d'acteurs ayant pour objet sa pratique professionnelle ou même bénévole, mis à part de manière résiduelle au sein du CRT. C'est en cela que la période ouverte par la révolution constitue un tournant majeur dans la structuration de la société civile autour de l'enjeu de l'immigration.

La révolution ayant fait émerger toute une série d'enjeux comme la surveillance démocratique, la visibilisation des minorités ethniques et sexuelles, la justice transitionnelle, les questions environnementales, etc., la société civile tunisienne a également ouvert son champ d'action à l'immigration. De fait, cette ouverture n'est pas uniquement symbolique, elle est aussi économique avec l'arrivée de plusieurs grands bailleurs de fonds européens, plus largement occidentaux, décidés à renforcer les

32. À ce sujet, lire le rapport du FTDES, Migrations non réglementaires, Tunisie 2017 [En ligne], 2017, https://ftdes.net/rapports/fr.omm3.2017.pdf.

33. Certaines victimes de ce naufrage faisaient partie de nos enquêtés. Des entretiens et des observations ont été réalisés dans les heures et les jours qui ont suivi l'accident avec des individus proches des victimes et des rescapés. 
«capacités» de la société civile tunisienne face aux «enjeux de la transition démocratique ». Cette réalité procède par rebond d'une vision purement occidentale et néolibérale de ce que doit être la «société civile $\gg^{34}$, à savoir un acteur tout à la fois légitimé par son rôle d'interlocuteur privilégié des institutions internationales, et légitimant pour les actions que celles-ci entreprennent sous son label.

Le domaine migratoire fait l'objet d'un particularisme en la matière, car le «renforcement des capacités», c'est-à-dire la diffusion et l'adoption par les acteurs visés d'une grille de lecture et de pratiques jugées comme «bonnes», se déploie alors selon une double logique. La première part du constat que l'État ne dispose d'aucune capacité ni expertise dans le domaine du migration management. C'est dans ce domaine que l'olM va faire preuve d'une action particulièrement importante. Son mandant consiste à «renforcer la capacité des gouvernements et des autres parties prenantes pertinentes à concevoir et à appliquer des politiques et des stratégies efficaces de gestion des migrations à l'échelle nationale $\gg^{35}$, via l'organisation d'ateliers de formation pour les fonctionnaires, de séminaires de recherche ou de réunions de coordination ${ }^{36}$, tout en continuant de proposer, par le biais de ces institutions publiques ou certaines ONG, son dispositif «d'aide au retour volontaire ${ }^{37}$. Ce soutien se traduit par le financement et la structuration d'institutions parapubliques comme I'Observatoire national de la migration, I'Instance nationale de lutte contre la traite, mais aussi par une aide à l'écriture de projets de loi comme celui de la lutte contre la traite des personnes ou celui contre les discriminations raciales. Enfin, I'OIM se trouve derrière la rédaction de la Stratégie nationale migratoire, sorte de document-cadre fixant des objectifs que l'État doit atteindre dans le domaine migratoire jusqu'en 2020. Cette démarche et les actions entreprises procèdent plus globalement de ce qui a été classiquement analysé dans les recherches sur cette organisation, à savoir la structuration via le système

34. Pour avoir une idée précise de la conception de la société civile par les institutions européennes, lire le Rapport de diagnostic sur la société civile tunisienne, dans le cadre de la mission de formulation, paru en mars 2012 : http://eeas.europa.eu/archives/delegations/tunisia/ documents/projets/rapportdiagnostic_stecivile_mars2012_fr.pdf.

35. Voir : https://tunisia.iom.int/content/dialogues-politiques-et-recherches.

36. Concernant les modes d'imposition du migration management en Tunisie, voir BARTELS, Inken, "Practices and Power of Knowledge Dissemination. International Organizations in the Externalization of Migration Management in Morocco and Tunisia", art. cité.

37. Voir : https://tunisia.iom.int/activities/aide-au-retour-volontaire-et-\%C3\%A0-la-r\%C3\%A 9int\%C3\%A9gration-avrr. 
de « partenariats », de réseaux décisionnels convertis à la « gestionalisation » et à la dépolitisation du fait migratoire ${ }^{38}$.

La deuxième logique part du constat indéniable que la société civile tunisienne ne dispose, elle non plus, d'aucune capacité ni connaissances sur la question migratoire, en termes de «bonnes pratiques », de « plaidoyer», ou d'accueil des migrants. Ainsi, une partie de la société civile commence alors à se former et se structurer autour de ce nouvel enjeu, notamment grâce à la création en 2012 de Tunisie terre d'asile, antenne tunisienne de France terre d'asile ${ }^{39}$. Son mandat, à la fois parallèle et coordonné à celui de l'OIM, consiste à «développer les capacités d'intervention opérationnelle et de plaidoyer de la société civile tunisienne dans le domaine de la défense des droits des migrants, des réfugiés et enfants non accompagnés ainsi que de participer à l'émergence d'un débat public démocratique, éclairé et apaisé sur les questions migratoires en Tunisie $\gg^{40}$, par le même mode opératoire que celui utilisé par I'olM. Ateliers de formation, organisation d'évènements culturels et diffusion d'appels à projets ayant pour objet cette « bonne gouvernance » ont ainsi constitué la matrice discursive autour de laquelle se sont nouées et formulées les problématiques tunisiennes autour de la question migratoire. L'élément le plus important étant peut-être que la question des droits des migrants, à savoir la simple normalisation de leur situation par l'attribution d'une carte de séjour, leur liberté de circuler, brille par son absence, en dehors d'actions visant à formuler des «plaidoyers ». Ainsi, l'élément central de cette « sensibilisation » concerne presque exclusivement l'identification des «besoins » des migrants, et la formulation de réponses à ces «besoins » par le secteur humanitaire et associatif.

L'année 2015 va constituer un second tournant concernant la question migratoire en Tunisie, car, à partir de ce socle sociologique et de ces réseaux socio-professionnels, le champ de l'assistance va se retrouver au centre d'une nouvelle vague de financements de la part des bailleurs de fonds européens, et notamment de l'Union européenne par le biais de son Fonds fiduciaire d'urgence, visant à « soutenir la mise en œuvre de la Stratégie nationale migratoire [...] avec les différents partenaires institu-

38. PÉCOUD, Antoine, "La bonne gouvernance des frontières?", dans Plein droit, $\mathrm{n}^{\circ} 87$, décembre 2010, pp. 24-27; ANDRIJASEVIC, Rutvica; WALTERS, William, "L'Organisation internationale pour les migrations et le gouvernement international des frontières", Cultures \& conflits, $n^{\circ} 4,2011$, pp. 13-43.

39. DINI, Sabine; GIUSA, Caterina, Externalising Migration Governance through Civil Society: Tunisia as a Case Study, New York: Palgrave MacMillan, à venir.

40. Voir : http://www.maison-migrations.tn/index.php/mdm/notre-projet. 
tionnels, organisationnels et de la société civile $\gg^{41}$. Le principal défi qu'ont posé ces nouveaux financements concernait leur répartition au sein de la configuration humanitaire et surtout les modalités de mise en œuvre des dispositifs qu'elle voulait mettre en place, Car, si l'objectif avoué de l'olm était de créer autour de la question migratoire un réseau associatif engagé dans «l'accompagnement» et «l'assistance 》 aux migrants, il ne s'agissait pas pour autant de financer n'importe quel projet et association. En recourant à un système d'appel à projets rigoureusement cadré, calqué sur un modèle entrepreneurial, I'olm a introduit un principe de distinction très structurant, désignant les bons acteurs et les mauvais acteurs de l'assistance. Ce principe de distinction, rarement assumé, se matérialise toutefois lorsqu'on analyse les conditions d'éligibilité des appels à projets mentionnant que «l'association postulante devra démontrer une expérience et une expertise technique dans le domaine de l'assistance psychosociale, éducative, culturelle, sportive et/ou artistique, auprès des populations vulnérables, et en particulier des migrants » alors que « les projets ayant un objectif idéologique et politique qui ne correspond pas à la stratégie, à la vision et aux principes de la mission de l'OIM $\gg^{42}$ seront eux considérés comme inéligibles.

Ainsi, la dépolitisation de l'action humanitaire dans le domaine migratoire se joue lors de la (re)distribution de la ressource économique essentielle à sa mise en œuvre. C'est dans ce cadre idéologique précis que doit se comprendre l'action des partenaires de l'olm en Tunisie, tous se retrouvant en fait récipiendaires et dépendants de ses financements, pour toute ou partie de leur action. Si la dimension partenariale constitue l'essentiel du «discours» produit sur les relations unissant I'OIM aux autres acteurs, notre analyse avance plutôt l'idée de rapports de domination symbolisés par le fait que l'olm détient le capital spécifique et symbolique du champ, nécessaire à la mise en œuvre de l'action humanitaire.

Plus globalement, il ressort de cet état de fait que la gestion sociale des populations migrantes, majoritairement africaines, c'est-à-dire la prise en charge médicale, psychologique, juridique, et même culturelle de leur quotidien, au sens anthropologique du terme, a fait l'objet d'une sous-traitance de l'État tunisien à l'Organisation internationale pour les migrations. Qu'ils soient arrivés de Libye ou directement en Tunisie, la participation des migrants aux différents dispositifs d'accompagnement

41. «La Tunisie, pays avec longue tradition migratoire, est depuis quelques années aussi un pays aussi de destination des migrants. Ainsi, les autorités tunisiennes sont en train d'élaborer une Stratégie nationale migratoire ». Source: https://ec.europa.eu/trustfundfor africa/region/north-africa/tunisia_en.

42. Lire par exemple la page 4 de cet appel à projet: https://tunisia.iom.int/sites/de fault/files/news/documents/AMI_RDPP\%20NA\%20DEV_15102018.pdf. 
humanitaire, articulés autour de «l'aide au retour volontaire », «la sensibilisation à l'immigration irrégulière», «l'intégration à la société tunisienne » ou «l'accès aux soins et à la santé » vont représenter la seule possibilité de visibilisation de leur existence dans la société, la «seule issue légale » à leur clandestinité. Le fait que les acteurs permettant cette visibilité soient issus de la société civile, donc porteurs d'un capital symbolique particulier, participe globalement au malentendu entourant les objectifs réels de ces dispositifs, à savoir, non pas une régularisation de leur situation et un combat pour leurs droits, mais bien une gestion de celle-ci dans un cadre juridique et politique bien défini. C'est en cela que l'on peut avancer l'idée que la gestion de l'immigration africaine en Tunisie constitue un «état d'exception » qui s'opère dans un cadre technique, financier, humain, opérationnel et idéologique créé sui generis, en dehors de l'action de l'État, pourtant acteur légitime et responsable de la prise en charge de ces populations.

\section{Conclusion}

II apparaît que la finalité des actions entreprises par le champ de l'assistance et de ses dispositifs n'est pas d'aboutir à la «régularisation » de cette immigration, à savoir à sa normalisation juridique et sociale, mais bien au contraire, elle semble participer de l'inertie entourant le statut de l'immigration africaine en Tunisie depuis sa relative reconnaissance depuis 2011, c'est-à-dire la poursuite d'une improbable acceptation et reconnaissance par l'État tunisien. Au-delà de cela, il apparaît évident que les moyens d'action mis en œuvre par la société civile légitimement reconnue dans la lutte pour les droits des migrants, comme le «plaidoyer», se heurtent très rapidement au périmètre d'action prédéfini par l'olM. II est clair aussi que ce périmètre d'action correspond à celui que l'État tolère sur son territoire, et à celui des bailleurs de fonds européens, dans les dispositifs qu'ils financent, ce qui constitue un périmètre « dépolitisé ». De cette manière, il nous est possible d'affirmer que l'objectif poursuivi par ces dispositifs humanitaires est bien «gestionnaire ». Ainsi, les questions centrales que pose la mise en œuvre de ces dispositifs se résument plutôt par, «comment faire de l'existence biologique et sociale de ces migrants l'objet d'une politique formalisant et guidant leur conduite dans la société tunisienne et sur le territoire tunisien? » L'objectif de la politique européo-tunisienne de gestion de l'immigration, si tant est qu'elle ait pu être formulée ainsi, n'est pas de chercher à « réprimer » par la violence la mobilité de ces personnes. Elle vise plutôt à encadrer et guider ces individus (et leur mobilité) vers des «choix » qui, formulés ainsi, correspondent à la fois aux intérêts objectifs de l'État tunisien, et 
de l'Union européenne, définis par la nécessité de garder sous contrôle une population par essence mobile et dont les projets ne trouvent pour limites que celles des réseaux migratoires auxquels ils sont reliés entre $i c i$ et là-bas. Le postulat théorique sous-jacent à cette recherche est que la gestion des populations ne s'opère que rarement dans une dimension purement «négative » comme dirait Michel Foucault ${ }^{43}$, ou pour reprendre l'expression de Michel Agier ${ }^{44}$, elle-même empruntée à Pierre Bourdieu ${ }^{45}$, par une unique «main droite » policière, répressive et néolibérale. Parce que cette «main droite » a déjà été partiellement analysée dans le contexte méditerranéen, nous avons pris le parti, dans cette contribution, d'éclairer les réalités de cette «main gauche», ce « pouvoir positif » qui ferait vivre les migrants à travers un ensemble de dispositifs humanitaires gérant les dimensions «clandestine » et «irrégulière » de leur existence. Le fait que cette main gauche soit incarnée et gérée par l'OIM et des ONG n'est pas étranger aux questionnements qui nous animent sur son orientation idéologique, c'est-à-dire non pas une assistance humaniste et désintéressée, mais une assistance dépolitisée construite dans la perspective de contrôler des populations par un ensemble de procédés et de pratiques, eux-mêmes fruits de la professionnalisation qu'a connue le monde de l'humanitaire ces vingt dernières années. Ainsi abordé, le paradoxe apparent de l'action de I'Union européenne en méditerranée centrale, qui financerait en même temps des acteurs humanitaires et sécuritaires, n'apparaît plus si paradoxal. Les traitements humanitaire et sécuritaire des populations migrantes semblent agir selon des modalités non pas antagonistes et opposées, mais bien complémentaires et réciproques et constituent aujourd'hui les deux faces d'un contrôle migratoire sans cesse perfectionné.

43. FOUCAULT, Michel, Naissance de la biopolitique : cours au Collège de France (1978-1979), Paris : Éd. Gallimard, 2004, 368 p.

44. AGIER, Michel, "La main gauche de I'Empire: ordre et désordres de l'humanitaire", Multitudes, vol. 11, $\mathrm{n}^{\circ} 1,2003$, pp. 67-77. 